\title{
Measurements and Diagnostics of Diamond Films and Coatings
}

\author{
Kazuhisa Miyoshi \\ National Aeronautics and Space Administration \\ Glenn Research Center \\ Cleveland, Ohio 44135-3191 USA \\ E-mail: miyoshi@grc.nasa.gov \\ Richard L.C. Wu \\ K Systems Corporation \\ 1522 Marsetta Drive \\ Beavercreek, Ohio 45432-2733 USA \\ E-mail: RLWU@aol.com
}

\begin{abstract}
The commercial potential of chemical-vapor-deposited (CVD) diamond films has been established and a number of applications have been identified through university, industry, and government research studies. This paper discusses the methodologies used for property measurement and diagnostic of CVD diamond films and coatings. Measurement and diagnostic techniques studied include scanning electron microscopy, transmission electron microscopy, atomic force microscopy, stylus profilometry, $x$-ray diffraction, electron diffraction, Raman spectroscopy, Rutherford backscattering, elastic recoil spectroscopy, and friction examination. Each measurement and diagnostic technique provides unique information. A combination of techniques can provide the technical information required to understand the quality and properties of CVD diamond films, which are important to their application in specific component systems and environments. In this study the combination of measurement and diagnostic techniques was successfully applied to correlate deposition parameters and resultant diamond film composition, crystallinity, grain size, surface roughness, and coefficient of friction.
\end{abstract}

Keywords: measurement; diagnostic methodology; chemical-vapor-deposited (CVD) diamond; films and coatings 


\subsection{Introduction}

A number of measurement and diagnostic techniques are now available for measuring the properties and composition of any solid material and its surface (1). Because the solid surface plays a crucial role in many mechanical, physical, and chemical processes (2), such as adhesion, oxidation, corrosion, erosion, wear, and friction, these measurement and diagnostic techniques have established their importance in a number of scientific, industrial, and commercial fields (1). During the last decade significant progress has taken place in the development of advanced surface films and coatings for engineering and biomedical applications. Their number is growing; some of the most exciting recent developments are superhard coatings and films, such as chemical-vapor-deposiled (CVD) diamond, diamondlike carbon (DLC), carbon nitride $\left(\mathrm{CN}_{\mathrm{x}}\right)$, and cubic boron nitride (c-BN) films (3).

The advantages and utility of CVD diamond as an industrial ceramic can only be realized if the quality, durability, and price are right (4). Until recently, this technology was of interest only to the academic and basic research communities. However, interest has grown because of advances made by leading CVD diamond suppliers $(4,5)$ :

1. The cost of CVD polycrystalline diamond deposition has been reduced below $\$ 5 /$ carat $\left(\$ 8 / \mathrm{cm}^{2}\right)$.

2. Production capacity has been installed.

3. Epitaxial growth of CVD single-crystal diamond has been achieved.

Thus, CVD diamond applications and business are an industrial reality.

At present CVD diamond is produced in the forms of coatings and wafers. CVD diamond film technology offers a broader technological potential than do natural and high-pressure synthetic diamonds because size, geometry, and eventually cost will not be as limiting. Now that they are cost effective, diamond coatings-with their extreme properties-can be used in a variety of applications. Diamond coatings can improve many surface properties of engineering substrate materials, including erosion, corrosion, and wear resistance (3). Examples of actual and potential applications, from microelectromechanical systems (MEMS) to environmentally durable barriers, of diamond coatings and related superhard coatings are described in reference 6 . For example, diamond coatings can be used as a chemical and mechanical barrier for Space Shuttle check valves, particularly the guide pins and seat assemblies (7). 
To achieve satisfactory quality and properties of diamond coatings and films, it is necessary to optimize deposition parameters by studying their effects on the physical, chemical, and structural properties of coatings and films. Deposition parameters must not only give the appropriate initial level of quality but must also provide durable and affordable coatings and films.

For a material to be recognized as diamond it must have all the following characteristics $(8,5)$ :

1. Crystalline diamond morphology and microstructure visible by optical or electron microscopy

2. Single-phase diamond crystalline structure detectable by $x$-ray or electron diffraction

3. Clear, sharp diamond peak at $1332 \mathrm{~cm}^{-1}$ in a Raman spectrum

4. Carbon content

5. Low coefficient of friction $(0.01$ to 0.05$)$ in air but high coefficient of friction $(>0.4)$ in ultrahigh vacuum

This paper deals with the application of measurement and diagnostic techniques in the technology of advanced CVD diamond films and related coatings. First, CVD diamond film deposition technology is briefly described. Then measurement and diagnostic techniques, including friction measurement, are highlighted in an important case study of microwave-plasma-assisted CVD diamond films. The work focused attention primarily on the nature, character, and quality of CVD diamond films. Some earlier data and experimental details on this research are given in the references (9-11).

\subsection{CVD Diamond Film Deposition Technology}

The basic reaction in the chemical vapor deposition of diamond is simple (8). It involves the decomposition of a hydrocarbon, such as methane, as follows:

$$
\mathrm{CH}_{4} \rightarrow(\text { Activation }) \rightarrow \mathrm{C}(\text { diamond })+2 \mathrm{H}_{2}
$$

The carbon species must be activated, since graphite is thermodynamically stable at low pressure and, without activation, only graphite would be formed. Activation is obtained by two basic methods: high temperature and plasma, both of which require a great deal of energy. 
Several chemical-vapor-deposition processes based on these two methods are presently in use.

The four most important activation methods at this time are

1. High-frequency, plasma glow discharge using the microwave and radiofrequency processes

2. Plasma arcing using the direct-current arc and radiofrequency arc processes

3. Thermal chemical vapor deposition using the hot-filament process

4. Combustion synthesis using an oxyacetylene torch

Plasma arcing and combustion synthesis have high substrate deposition rates $(4,8)$. For example, the mass deposition rate using a direct-current arc jet was 20 carats/hr in 1997 (4). Progress in directcurrent arc jet deposition has advanced to the point that the $\$ 5 / \mathrm{carat}\left(\$ 8 / \mathrm{cm}^{2}\right)$ barrier has been breached (4). In 1997 diamond made by direct-current arc jet was available at $\$ 8 / \mathrm{cm}^{2}\left(\$ 50 / \mathrm{in} .{ }^{2}\right)$.

Diamond has been deposited from a large variety of precursors, including methane, aliphatic and aromatic hydrocarbons, alcohols, ketones, and solid polymers such as polyethylene, polypropylene, and polystyrene (8). These substances generally decompose into two stable primary species: the methyl radicals $\left(\mathrm{CH}_{3}\right)$ and acetylene $\left(\mathrm{C}_{2} \mathrm{H}_{4}\right)$. The radical is the key compound in generating the growth of CVD diamond.

\subsection{Measurement and Diagnostic of CVD Diamond}

A variety of techniques can be used to characterize CVD diamond films. Measurement and diagnostic techniques used in this investigation include

1. Scanning electron microscopy and transmission electron microscopy (SEM and TEM), to determine surface morphology, microstructure, and grain size

2. Surface profilometry and atomic force microscopy (AFM), to measure surface roughness and to determine surface morphology

3. Rutherford backscattering (RBS) and elastic recoil spectroscopy (ERS), to determine the composition (including hydrogen)

4. Raman spectroscopy, to characterize the atomic bonding state and quality of diamond

5. X-ray diffraction (XRD), to determine the crystal orientation of diamond

6. Friction examination, to determine the coefficient of friction and surface properties 
The following case studies (9-11) focus attention primarily on microwave-plasma-assisted CVD diamond films.

\subsection{Electron Microscopy, Stylus Profilometry, and Atomic Force Microscopy}

Transmission electron microscopy offers two methods of specimen observation: image mode and diffraction mode (12). In image mode, analysis of transmitted electron images yields information both about atomic structure and about defects present in the material. In diffraction mode, an electron diffraction pattern is obtained from the specimen area illuminated by the electron beam. The electrun diffraction pattern is entirely equivalent to an $x$-ray diffraction pattern.

Scanning electron microscopy (with energy dispersive spectroscopy) is the most useful tool when the researcher needs not only morphological and topographical information about surfaces but also information concerning the composition of near-surface regions of the material. Although diamond is an insulator, it can be studied by using low primary electron beam voltages ( $5 \mathrm{keV}$ or less) if one is willing to compromise image resolution to some extent. If the diamond is coated with a thin conducting film (10 to $20 \mathrm{~nm}$ thick) of carbon, gold, or some other metal, the coated diamond can be studied with an image resolution of 1 to $50 \mathrm{~nm}$.

The stylus (mechanical) profilometer is today commonly used for measuring surface roughness and analyzing topography. A diamond stylus with a tip radius of a few micrometers moves up and down as it is dragged across a specimen surface. This up-and-down motion effectively replicates the surface topography.

The grain size, surface morphology, and surface roughness of a microwave-plasma-assisted CVD diamond film can be controlled by varying the deposition parameters, such as gas-phase chemistry parameters and temperatures (e.g., Table 1). The grain size and surface roughness data were obtained by using TEM and stylus profilometry. The CVD diamond films referred to in Table 1 can be divided into three groups by grain size: fine, medium, and coarse grain. The grain sizes of the fine-grain diamond films were determined from bright- and dark-field electron photomicrographs to be between 20 and $100 \mathrm{~nm}$. The medium- and coarse-grain diamond films have grain sizes estimated at 1000 to $1500 \mathrm{~nm}$ and $3300 \mathrm{~nm}$, respectively. The average surface roughness of the diamond films measured by a surface profilometer increased as the grain size increased, as shown in Fig. 1. Figure 2 shows scanning electron photomicrographs of fine-, medium-, and coarse-grain diamond films. Triangular 
crystalline facets typical of diamond are clearly evident on the surfaces of the medium- and coarsegrain films.

In an atomic force microscope, to obtain topographical information, a probe tip traverses across a diamond surface and senses the force of interaction between itself and the diamond surface. By monitoring the tip deflection necessary to maintain a constant interacting force, surface topographical data can be obtained on a nanometer scale. Figure 3 is an AFM image of a fine-grain CVD diamond film. The surface has a granulated or spherulitic morphology (i.e., it contains spherical asperities of different sizes).

\subsection{X-Ray Diffraction and Electron Diffraction}

$\mathrm{X}$-ray diffraction is a powerful technique used to uniquely identify the crystalline phases present in materials and to measure the structural properties of these phases (12). Although $\mathrm{x}$-ray diffraction is not inherently a surface diagnostic technique, it offers unparalleled accuracy in the measurement of atomic spacing. XRD was used to determine the structure and crystal orientation of the CVD diamond films (10). Typical x-ray diffraction patterns for the fine- and medium-grain diamond films (Fig. 4) show peaks representing only the diamond film and the silicon substrate. Diffraction peaks corresponding to the $(111\},\{220\},\{311\}$, and $\{400\}$ planes, reflective of diamond, are clearly evident. The intensity ratios $I\{220\} / I(111\}$ were calculated from the $\mathrm{x}$-ray diffraction patterns for these films and found to be 1.3 and 0.04 , respectively. The powder diffraction pattern of diamond with random crystal orientation (ASTM 6-0675) gives $l(220) / I(I I I)=0.27$. Thus, most crystallites in the fine-grain diamond films are oriented along the $\langle 110\rangle$ direction, whereas most crystallites in the medium-grain diamond films are oriented in the $\langle 111\rangle$ direction. The well-formed triangular facets observed in SEM photomicrographs of medium- and coarse-grain diamond films confirm the $\langle 111\rangle$ crystal orientation.

Figure 5 presents a TEM selected-area diffraction pattern (SAD), a TEM bright-field photomicrograph, and a TEM dark-field photomicrograph of a free-standing, fine-grain CVD diamond film (9). Diffraction rings and dots can be observed in Fig. 5(a). The d spacings of the diffraction rings were calculated by using an aluminum SAD as a calibration standard and were found to match well with the known diamond d spacings. No evidence of nondiamond carbon was found in the SAD. This observation indicates that the nondiamond concentration in the diamond film was extremely small. Careful observation of Fig. 5(b) revealed various nuclei-like regions marked N. Diamond grains are 
distributed radially outward from these nuclei. A grain boundary is formed where the grains from various nuclei meet. As previously mentioned, the grain sizes of the fine-grain CVD diamond films estimated from the bright- and dark-field photomicrographs varied from 20 to $100 \mathrm{~nm}$.

\subsection{Raman Spectroscopy}

Raman spectroscopy is primarily a structural characterization tool (12). Raman spectra are more sensitive to the length and strength of bonds and to their arrangement in a material than to chemical composition. Raman spectra of crystals likewise reflect the details of defects and disorder rather than trace impurities and related chemical imperfections. The laser-optical Ranan technique can determine with great confidence the atomic bonding states of the carbon atoms $\left(\mathrm{sp}^{2}\right.$ for graphite or $\mathrm{sp}^{3}$ for diamond) because of their different vibrational modes (13). Raman spectra result from the inelastic scattering of optical photons by lattice vibration phonons.

Typical Raman spectra of the fine- and medium-grain diamond films (Fig. 6) show one Raman band centered at $1332 \mathrm{~cm}^{-1}$ and one centered around $1530 \mathrm{~cm}^{-1}$. The sharp peak at $1332 \mathrm{~cm}^{-1}$ is characteristic of the $\mathrm{sp}^{3}$ bonding of the diamond form of carbon in the film. The very broad peak centered around $1530 \mathrm{~cm}^{-1}$ is attributed to the $\mathrm{sp}^{2}$ bonding of the nondiamond forms of carbon (graphite and other carbon) (14-16).

More diamond is produced in larger grain CVD diamond films (e.g., Fig. 6(b)) than in fine-grain films, as is evident from the relative intensities of the diamond and nondiamond carbon Raman bands (10). However, the ratio of the intensities of the Raman responses at $1332 \mathrm{~cm}^{-1}$ and centered around $1530 \mathrm{~cm}^{-1}$ does not indicate the ratio of diamond to nondiamond carbon present in a particular film, since the Raman technique is approximately 50 times more sensitive to $\mathrm{sp}^{2}$-bonded (nondiamond) carbon than to $\mathrm{sp}^{3}$-bonded (diamond) carbon (15). Thus, the peak centered around $1530 \mathrm{~cm}^{-1}$ for each film represents a much smaller amount of nondiamond carbon in these diamond films than appears at first glance.

\subsection{Rutherford Backscattering Spectroscopy and Elastic Recoil Spectroscopy}

Rutherford backscattering is a nondestructive, quantitative depth profiling of thin-film compositions and structures, crystallinity, dopants, and impurities (12). Elastic recoil spectroscopy (also called hydrogen forward scattering or proton recoil detection) is the simplest ion beam technique 
for hydrogen profiling and determines hydrogen concentrations in thin films. In combination with RBS analysis of the same sample ERS provides concentration profiles and complete compositional analysis of the near-surface regions of the sample material.

Figures 7 and 8 present RBS and ERS spectra, respectively, of a fine-grain CVD diamond film (9). Besides carbon from the diamond film and silicon from the silicon substrate, no other elements appear in the RBS spectrum. From both spectra it was estimated that the fine-grain diamond film consisted of 97.5 at.\% carbon and 2.5 at.\% hydrogen. (In contrast, the medium-grain diamond films contained less than 1 at.\% hydrogen (10).) It was also demonstrated that both carbon and hydrogen are uniformly distributed in the fine-grain film from the top of the surface to the silicon substrate.

RBS analytical results can also be used to determine diamond film thickness. Figure 7 presents a simulated RBS spectrum of the diamond film containing a carbon-to-hydrogen ratio $(\mathrm{C} / \mathrm{H})$ of $97.5 / 2.5$ obtained by using the RUMP computer code (17). In the computer program the film thickness of the diamond film is taken as a variable. This thickness was obtained from the close match between the observed and simulated RBS, as shown in Fig. 7, and is $1.5 \mu \mathrm{m}$ at the center of the substrate. The deposition rate was estimated to be $0.14 \mu \mathrm{m} / \mathrm{hr}$.

\subsection{Friction Measurement}

The classical Bowden and Tabor model for sliding friction of materials in contact, in its simple form, assumes that the friction force arises from two contributing sources (18). First, an adhesion force is developed at the real area of contact between the surfaces, arising from the attractive forces between the contacting surfaces. Second, a deformation force is needed to plow or cut the asperities of the harder surface through the softer. The resultant friction force is the sum of the two contributing sources: friction due to adhesion and friction due to deformation and/or fracture. When a smooth flat is brought into contact with a smooth spherical surface of the same or softer material, the plowing or cutting contribution in friction can be neglected. In this situation, as is well known, diamond is one of the slipperiest materials and is similar to polytetrafluoroethylene in air. The coefficient of friction is between 0.01 and 0.05 in air.

The friction and wear properties of CVD diamond films are similar to those of natural and synthetic diamond. The coefficient of friction and wear resistance of CVD diamond are generally superior in the atmosphere. However, the environment to which a CVD diamond film is exposed can markedly affect its friction and wear behavior. For example, the coefficient of friction is greater than 
0.4 in ultrahigh vacuum. Thus, the behavior varies with the environment, giving CVD films a Jekylland-Hyde character (10).

3.5.1 Humid air and dry nitrogen environments.-When the fine-, medium-, and coarse-grain CVD diamond films characterized in previous sections were brought into contact with a natural diamond pin in reciprocating sliding motion in air and in nitrogen, the coefficients of friction varied as the pin traveled back and forth (reciprocating motion), retracing its tracks on the diamond films (Fig. 9). Both in humid air at a relative humidity of $40 \%$ and in dry nitrogen, abrasion occurred and dominated the friction and wear behavior. The bulk natural diamond pin tended to dig into the surface of diamond films during sliding and produce a wear track (groove). SEM observations of the diamond films indicated that small fragments chipped off their surfaces. When abrasive interactions between the diamond pin surface and the initially sharp tips of asperities on the diamond film surfaces were strong, the friction was high (points A in Fig. 9). The surface roughness of diamond films can have an appreciable influence on their initial friction (i.e., the greater the initial surface roughness, the higher the initial coefficient of friction, Fig. 10). Similar frictional results have also been found by other workers on single-crystal diamonds (19) and on diamond coatings (20-22).

As sliding continued and the pin passed repeatedly over the same track, the coefficient of friction was appreciably affected by the wear on the diamond films (Fig. 9) (i.e., a blunting of the asperity tips). When repeated sliding produced a smooth groove or a groove with blunted asperities on the diamond surface (Fig. 11), the coefficient of friction (now due only to adhesion) was low, and the initial surface roughness effect became negligible. Therefore, the equilibrium coefficient of friction was independent of the initial surface roughness of the diamond film (Fig. 10(b)).

The generally accepted wear mechanism for diamonds is that of small fragments chipping off the surface $(23,10)$. This mechanism agrees with the wear of diamond films. The wear rate depends on the initial surface roughness of the diamond films (Fig. 12), increasing markedly with an increase in initial surface roughness. The wear rates of the diamond films in humid air and in dry nitrogen are comparable to the wear rates of single-crystal diamonds and diamond films investigated by other workers $(20,24,11)$.

3.5.2 Ultra-high-vacuum environment.-When the fine-, medium-, and coarse-grain diamond films were brought into contact with a natural diamond pin in unidirectional pin-on-disk sliding motion in vacuum, the coefficients of friction were high and varied with the number of passes (10). In vacuum, as in humid air and in dry nitrogen, the bulk natural diamond pin dug into the surfaces of the diamond films during sliding and produced a wear track (groove, Fig. 13). The groove surface was 
generally smoother than the original surface of the diamond films. Further analysis of the grooves by scanning electron microscopy revealed that the tips of the diamond coating asperities were worn smooth and that the gaps between asperities were filled by debris.

The coefficient of friction increased with an increase in the number of passes (Fig. 14), just the opposite of what occurred in humid air and in dry nitrogen. Further, the initial surface roughness of the diamond film had no effect on friction. These results led us to ask the following questions: What factors determine friction behavior? Have dangling bonds, each of which carries an unpaired electron spin, been exposed during sliding and played a role in the friction behavior? Which is more important for diamond surfaces in vacuum: abrasion or adhesion?

A contaminant surface film may be removed by repeatedly sliding a diamond pin over the same track in ultrahigh vacuum (11). When some contaminant surface film was removed from the contact area of diamond films by sliding action, stronger interfacial adhesion resulted between the diamond pin and the diamond films, raising the coefficient of friction as shown in Fig. 14. The atoms exposed at the free surfaces possibly possessed a dangling bond, thus retaining such a high friction.

The friction results shown in Fig. 14 agree with other researchers' results for single-crystal diamond rubbing against diamond and for CVD diamond sliding against CVD diamond in vacuum $(25,26)$. Bowden and Hanwell $(26)$ observed an initial coefficient of friction of 0.1 in vacuum ( $93 \mathrm{nPa}$ ) for diamond on diamond; within several hundred passes, however, the coefficient of friction rose rapidly to 0.9 and remained constant. Dugger, Peebles, and Pope (27) also found that the coefficient of friction rose to 0.47 when CVD diamond slid against itself in vacuum $(<0.6 \mu \mathrm{Pa})$. In both cases the increase in friction was attributed to cleaning the adsorbed contaminants from the surface by rubbing or sliding in vacuum at room temperature.

When sliding continued, the wear dulled the asperity tips of the diamond and enlarged the contact area in the wear track, thereby causing friction to rise. The increase in equilibrium friction that resulted from cleaning off the contaminant surface film by sliding and from enlarging the contact area was greater than the corresponding decrease in abrasion and friction that resulted from blunting the tips of surface asperities. This relationship is brought out clearly in Fig. 15, where the equilibrium coefficients of friction ( 1.5 to 1.8 ) are greater than the initial coefficients of friction (1.1 to 1.3) regardless of the initial surface roughness of the diamond films. Here again, to create new surfaces by wear, great adhesion and friction clearly were involved. In vacuum, therefore, the friction arises primarily from adhesion between the sliding surfaces of the diamond pin and the diamond films. 
The wear rates of the diamond films in ultrahigh vacuum (Fig. 16) depended on the initial surface roughness of the diamond films, generally increasing with an increase in initial surface roughness. The wear rates of the diamond films were considerably higher in ultrahigh vacuum than in humid air or in dry nitrogen (Fig. 12). Obviously, under these vacuum conditions adhesion between the sliding surfaces of the diamond pin and the diamond film plays an important role in the higher wear process.

Thus, under vacuum conditions it is adhesion between the sliding surfaces of the diamond pin and the diamond films (due to the highly clean state) and the possible presence of dangling bonds that play a significant role in the friction and wear process. The surface roughness of the diamond films does not have much influence on the friction of diamond films in ultrahigh vacuum.

\subsection{Concluding Remarks}

University, industry, and government research studies have established the commercial potential of chemical-vapor-deposited (CVD) diamond films and have identified a number of applications. However, the technical application and utility of CVD diamond in specific component systems and environments can be achieved only if the quality, durability, and price are right. To achieve satisfactory coatings and films, it is necessary to optimize the deposition parameters. To understand the benefits provided by deposition parameters, and ultimately to choose better parameters and thus improve film and coating performance, a variety of measurement and diagnostic techniques must be used to investigate the physical, chemical, and structural changes in films and coatings produced by the deposition parameters.

The use of measurement and diagnostic techniques, including friction examination, was highlighted in the important case study of microwave-plasma-assisted, chemical-vapor-deposited diamond films. Each measurement and diagnostic technique provides unique information. It should be possible to coordinate the different pieces of information provided by these techniques into a coherent self-consistent description of the surface and bulk properties. In this case study a combination of measurement and diagnostic techniques was successfully applied to correlate deposition parameters and resultant diamond film composition, crystallinity, grain size, surface roughness, and coefficient of friction. These methodologies have contributed significantly to our understanding of the properties of diamond films and their surfaces. 


\section{References}

1. Miyoshi, K. and Chung, Y.W., Surface Diagnostics in Tribology: Fundamental Principles and Applications, World Scientific Publishing Co., River Edge, New Jersey, 1993.

2. Jost, H.P., Tribology-origin and future, Wear, 1990, 136, 1-17.

3. Molloy, A.D. and Dionne, A.M. (eds.), Wear and Superhard Coatings 1998, Gorham Advanced Materials, Inc., Gorham, Maine, 1998.

4. Windischmann, H., Stress issues in wafer-scale CVD diamond fabrication, Book of Abstracts, American Vacuum Society, 1998, p. 124; and personal communication.

5. Miyoshi, K., Chemical-vapor-deposited diamond films, NASA/TM-1999-107249, Chapt. 9, Feb. 1999.

6. Miyoshi, K., Murakawa, M., Watanabe, S., Takeuchi, S., and Wu, R.L.C., Tribological characteristics and applications of superhard coatings: CVD diamond, DLC, and c-BN, NASA/TM-1999-209189, Aug. 1999.

7. Miyoshi, K., Aerospace mechanisms and tribology technology: case studies, NASA/TM-1999-107249, Chapt. 7, Aug. 1999.

8. Pierson, H.O., Handbook of Carbon, Graphite, Diamond and Fullerenes, Noyes Publications, Park Ridge, New Jersey, 1993.

9. Wu, R.L.C., Rai, A.K., Garscadden, A., Kee, P., Desai, H.D., and Miyoshi, K., Synthesis and characterization of fine grain diamond films, Journal of Applied Physics, July 1992, 2(1), 110-116.

10. Miyoshi, K., Friction and wear of plasma-deposited diamond films, Journal of Applied Physics, Oct. 1993, $74(7), 4446-4454$.

11. Miyoshi, K., Wu, R.L.C., and Garscadden, A., Friction and wear of diamond and diamond-like carbon coatings, Surface Coating Technology, 1992, 54/55, 428-434.

12. Brundle, C.R., Evans, C.A., Jr., and Wilson, S., Encyclopedia of Materials Characterization, ButterworthHeinemann, Boston, Massachusetts, 1992.

13. Nemanich, R.J., Glass, J.T., Lucovsky, G., and Shroder, R.E., Raman scattering characterization of carbon bonding in diamond and diamondlike thin films, Journal of Vacuum Science Technology A, 1988, 6(3), 1783-1787.

14. Cheng, J.J., Mautei, T.D., Vuppulahadium, R., and Jackson, H.E., Effects of oxygen and pressure on diamond synthesis in a magnetoactive microwave discharge, Journal of Applied Physics, 1992, 71 (6), 2918-2923.

15. Kobashi, K., Nishinura, K., Kawate, V., and Horiuchi, T., Synthesis of diamonds by use of microwave plasma chemical vapor deposition: morphology and growth of diamond film, Physics Review, 1988, B38(6), $4067-4084$. 
16. Wada, N. and Solin, S.A., Raman efficiency measurements of graphite (and Si and Ge), Physica B\&C, 198I, $105,353-356$.

17. Doolittle, L.R., Algorithms for the rapid simulation of Rutherford backscattering spectra, Nuclear Instrumentation Methods, 1985, B9, 344-351.

18. Bowden, F.P. and Tabor, D., The Friction and Lubrication of Solids, Clarendon Press, Oxford, England, 1958.

19. Casey, M. and Wilks, J., The friction of diamond sliding on polished cube faces of diamond, Journal of Applied Physics D, 1973, 6(15), 1772-1781.

20. Hayward, I.P., Friction and wear properties of diamond and diamond coatings, Surface Coating Technology, $1991,49,554-559$.

21. Hayward, I.P. and Singer, I.L., Tribological behavior of diamond coatings, in Second International Conference on New Diamond Science and Technology, Materials Research Society, Pittsburgh, Pennsylvania, $1991,785-789$.

22. Hayward, I.P., Singer, I.L., and Seitzman, L.E., Effect of roughness on the friction of diamond on CVD diamond coatings, Wear, 1992, 157, 215-227.

23. Tabor, D., Adhesion and Friction, in The Properties of Diamond, ed. J.E. Field, Academic Press, New York, 1979, pp. 325-350.

24. Crompton, D., Hirst, W., and Howse, M.G.W., The wear of diamond, Proceedings of the Royal Society London Series $A, 1973,333,435-454$.

25. Feng, Z., Tzeng, Y., and Field, J.E., Friction of diamond on diamond in ultra-high-vacuum and low-pressure environments, Journal of Applied Physics D, 1992, 25(10), 1418-1424.

26. Bowden F.P. and Hanwell, A.E., The friction of clean crystal surfaces, Proceedings of the Royal Society London Series A, 1966, 295, 233-243.

27. Dugger, M.T., Peebles, D.E., and Pope, L.E., Counterface material and ambient atmosphere: role in the tribological performance of diamond films, in Surface Science Investigations in Tribology, eds. Y.-W. Chung, A.M. Homola, and G.B. Street, American Chemical Society, Washington, DC, 1992, pp. 72-102. 


\section{Figure Legends:}

Figure 1.-Surface roughness as function of grain size for diamond films.

Figure 2.-Scanning electron micrographs of diamond films. (a) Fine-grain (20 to $100 \mathrm{~nm}$ ) diamond film on $\{100\}$ silicon substrate; rms surface roughness, $15 \mathrm{~nm}$. (b) Medium-grain (1100 nm) diamond film on $\{100\}$ silicon substrate; rms surface roughness, $63 \mathrm{~nm}$. (c) Coarse-grain (3300 nm) diamond film on $\alpha$-silicon carbide substrate; rms surface roughness, $160 \mathrm{~nm}$.

Figure 3.-Atomic force micrograph of chemical-vapor-deposited, fine-grain diamond film on mirror-polished silicon substrate.

Figure 4.-X-ray diffraction patterms of diamond films. (a) Fine-grain (20 to $100 \mathrm{~nm})$ diamond film on $\{100\}$ silicon substrate. (b) Medium-grain (1100 nm) diamond film on (100) silicon substrate.

Figure 5.-Free-standing diamond films. (a) Selected-area diffraction pattern. (b) Bright-field TEM. (c) Dark-field TEM.

Figure 6.-Raman spectra of diamond films. (a) Fine-grain (20 to $100 \mathrm{~nm}$ ) diamond film on (100\} silicon substrate. (b) Medium-grain (1100 nm) diamond film on (100) silicon substrate.

Figure 7.-Rutherford backscattering spectrum of fine-grain diamond film on silicon substrate. (Simulation curve was calculated by using computer code RUMP.)

Figure 8.-Elastic recoil spectrum of fine-grain diamond on silicon substrate.

Figure 9.-Coefficient of friction as function of number of passes of bulk diamond pin in contact with fine-, medium-, and coarse-grain diamond films (a) in humid air (approx. $40 \%$ relative humidity) and (b) in dry nitrogen. 
Figure 10.- Coefficient of friction as function of initial surface roughness of diamond films in humid air (approx. $40 \%$ relative humidity) and in dry nitrogen. (a) Initial coefficients of friction. (b) Equilibrium coefficients of friction.

Figure 11.-Wear tracks (grooves) on diamond films after 30000 passes of bulk diamond in dry nitrogen. (a) Fine-grain (20 to $100 \mathrm{~nm}$ ) diamond film on $\{100\}$ silicon substrate; rms surface roughness, $15 \mathrm{~nm}$. (b) Mediumgrain (1100 nm) diamond film on $\{100\}$ silicon substrate; rms surface roughness, $63 \mathrm{~nm}$. (c) Coarse-grain (3300 $\mathrm{nm}$ ) diamond film on $\alpha$-silicon carbide substrate; rms surface roughness, $160 \mathrm{~nm}$.

Figure 12.-Wear rate of diamond films as function of diamond surface roughness in humid air and in dry nitrogen.

Figure 13.- Wear tracks (grooves) on diamond films after 100 passes of bulk diamond in ultrahigh vacuum. (a) Fine-grain (20 to $100 \mathrm{~nm}$ ) diamond film on (100) silicon substrate; rms surface roughness, $15 \mathrm{~nm}$. (b) Mediumgrain $(1000 \mathrm{~nm})$ diamond film on (100) silicon substrate; rms surface roughness, $52 \mathrm{~nm}$. (c) Coarse-grain (1500 $\mathrm{nm}$ ) diamond film on $\alpha$-silicon carbide substrate; rms surface roughness, $92 \mathrm{~nm}$.

Figure 14.-Typical friction trace for bulk diamond pin in contact with diamond film on $\alpha$-silicon carbide substrate in ultrahigh vacuum (initial coefficient of friction, $\mu_{l}$; equilibrium coefficient of friction, $\mu_{r}$ )

Figure 15.-Initial $\left(\mu_{l}\right)$ and equilibrium $\left(\mu_{t}\right)$ coefficients of friction as function of initial surface roughness of diamond films in ultrahigh vacuum.

Figure 16.- Wear rate as function of initial surface roughness of diamond films in ultrahigh vacuum. 
TABLE 1.-DEPOSITION CONDITIONS FOR DIAMOND FILMS OF VARIOUS GRAIN SIZES

\begin{tabular}{|l|r|r|r|r|r|r|r|}
\hline \multirow{2}{*}{ Condition } & \multicolumn{7}{|c|}{ Substrate $^{\mathrm{a}}$} \\
\cline { 2 - 8 } & $\mathrm{Si} \mathrm{(100)}$ & $\mathrm{Si}(100)$ & $\alpha$ - $\mathrm{SiC}$ & $\alpha$-SiC & $\alpha$-SiC & $\mathrm{Si}_{3} \mathrm{~N}_{4}$ & $\mathrm{Si}_{3} \mathrm{~N}_{4}$ \\
\hline Deposition temperature, ${ }^{\circ} \mathrm{C}$ & $860 \pm 20$ & $1015 \pm 50$ & $1015 \pm 50$ & $965 \pm 50$ & $860 \pm 20$ & $965 \pm 50$ & $860 \pm 20$ \\
\hline Gaseous flow rate, $\mathrm{cm}^{3} / \mathrm{min}:$ & & & & & & & \\
$\mathrm{CH}_{4}$ & 4 & 3.5 & 3.5 & 3.5 & 4 & 3.5 & 4 \\
$\mathrm{H}_{2}$ & 395 & 500 & 500 & 500 & 395 & 5800 & 395 \\
$\mathrm{O}_{2}$ & 1 & 0 & 0 & 0 & 1 & 0 & 1 \\
\hline Pressure, torr & 5 & 40 & 40 & 40 & 5 & 40 & 5 \\
\hline Microwave power, $\mathrm{W}$ & 500 & 100 & 1000 & 1000 & 500 & 1000 & 1500 \\
\hline Deposition time, $\mathrm{hr}$ & 10.5 & 140 & 14 & 22 & 21 & 22 & 521 \\
\hline Thickness, nm & 1000 & 4200 & 5000 & 8000 & 1000 & 7000 & 800 \\
\hline Grain size, nm & $20-100$ & 1100 & 3300 & 1500 & $22-100$ & 1000 & $22-100$ \\
\hline Surface roughness rms, $\mathrm{nm}$ & 15 & 63 & 160 & 92 & 50 & 52 & 35 \\
\hline
\end{tabular}

${ }^{2}$ Scratched with 0.5-mm diamond paste. 


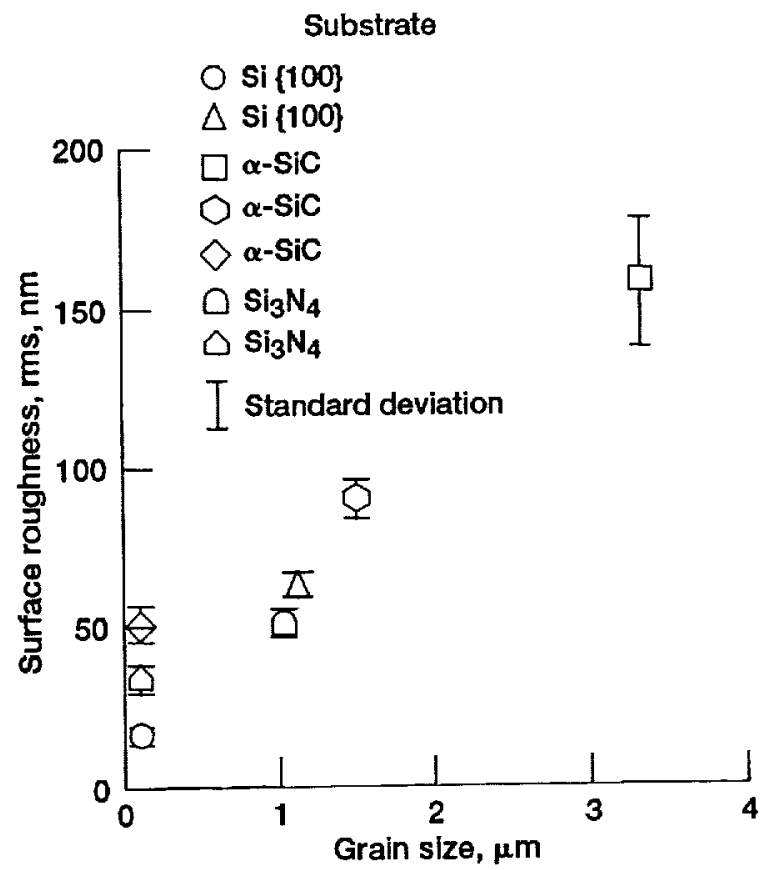

Figure 1.-Surface roughness as function of grain size for diamond films. 

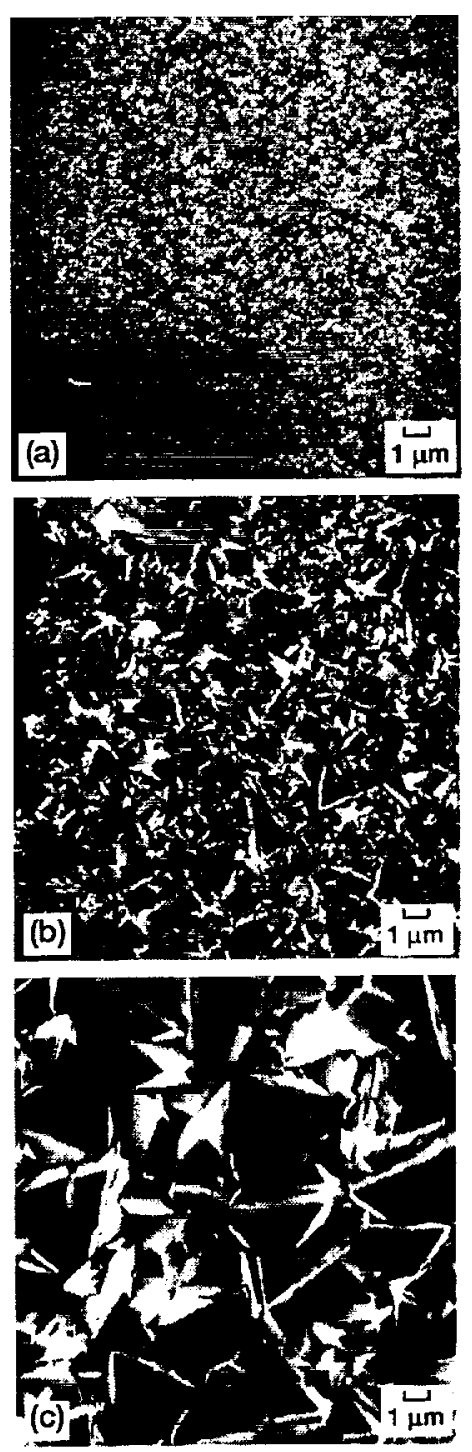

Figure 2.-Scanning electron micrographs of diamond films. (a) Fine-grain (20 to $100 \mathrm{~nm}$ ) dlamond film on $\{100\}$ silicon substrate; rms surface roughness, $15 \mathrm{~nm}$. (b) Medium-grain (1100 nm) diamond film on $\{100\}$ silicon substrate; $m$ s surface roughness, $63 \mathrm{~nm}$. (c) Coarse-grain $(3300 \mathrm{~nm})$ diamond film on $\{100\} \alpha$-silicon carbide substrate; rms surface roughness, $160 \mathrm{~nm}$. 

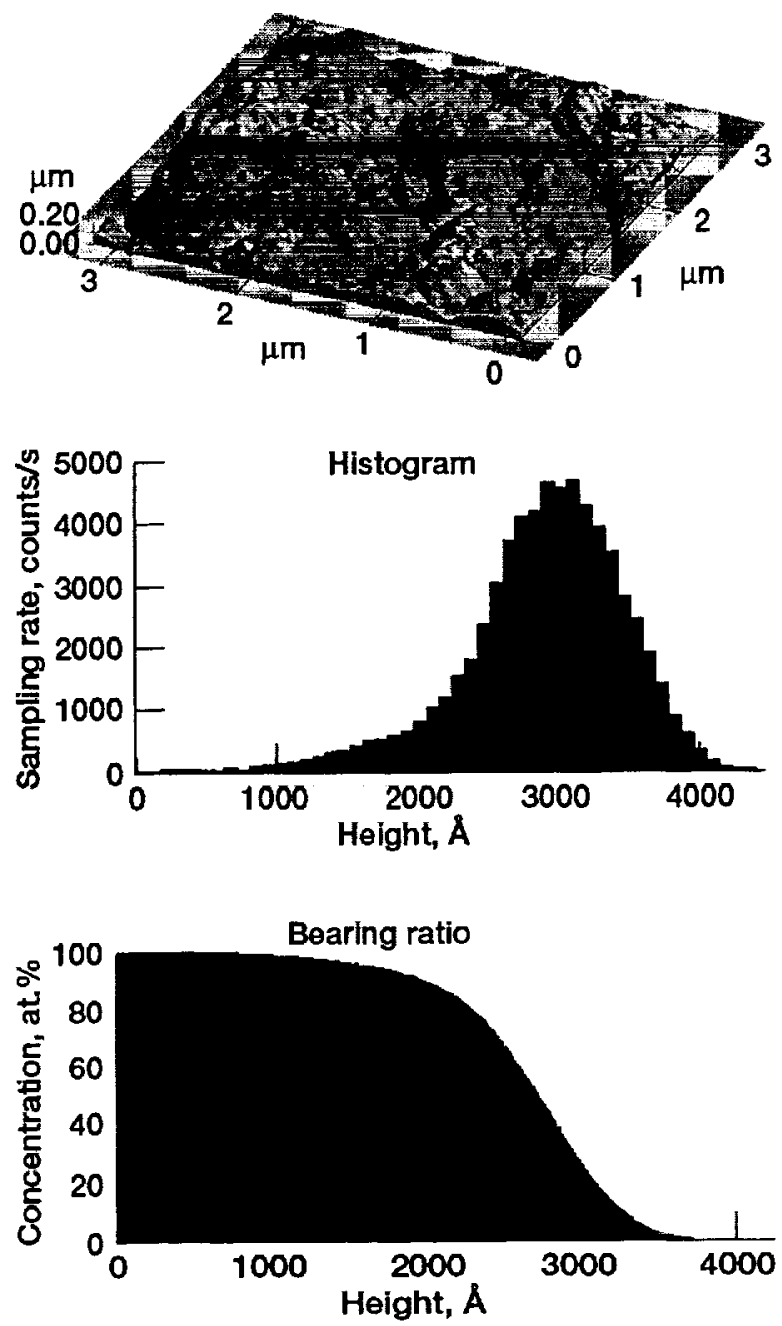

Figure 3.-Atomic force micrograph of chemicalvapor-deposited, fine-grain diamond film on mirror-polished silicon substrate. 

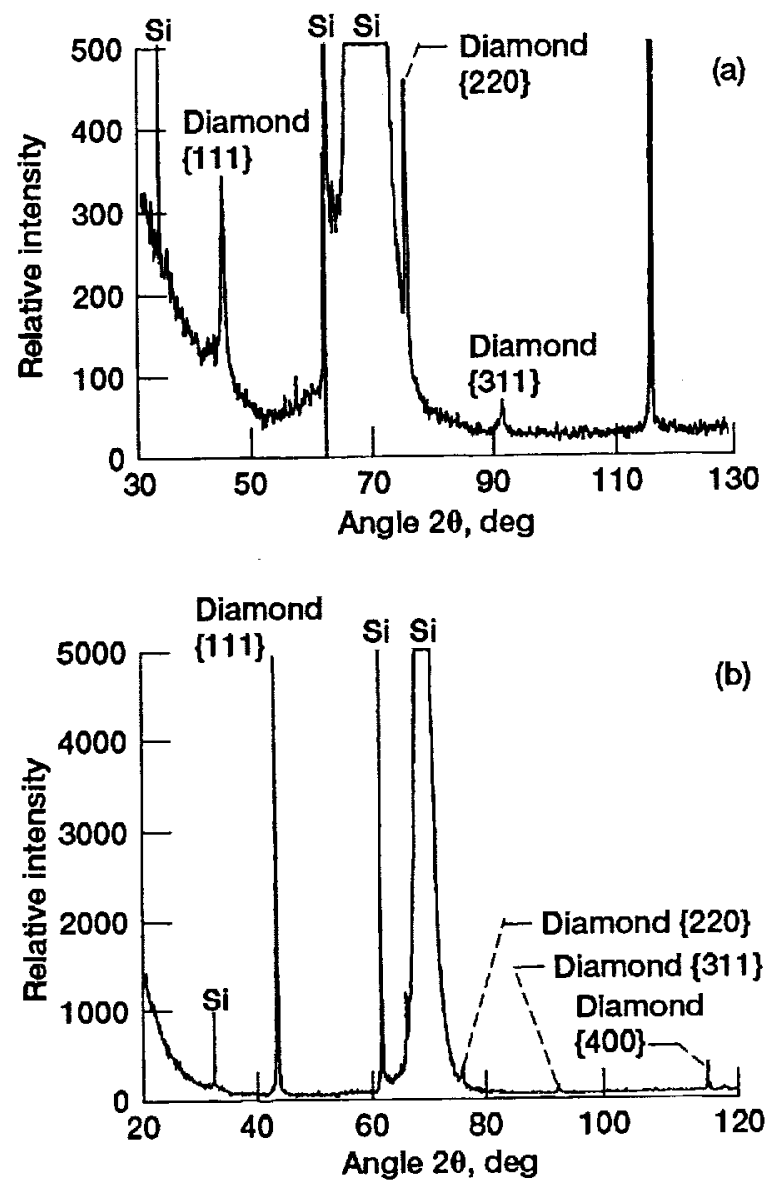

Figure 4.-X-ray diffraction patterns of diamond films. (a) Fine-grain $(20$ to $100 \mathrm{~nm})$ diamond film on $\{100\}$ silicon substrate. (b) Medium-grain $(1100 \mathrm{~nm})$ diamond film on $\{100\}$ silicon substrate. 

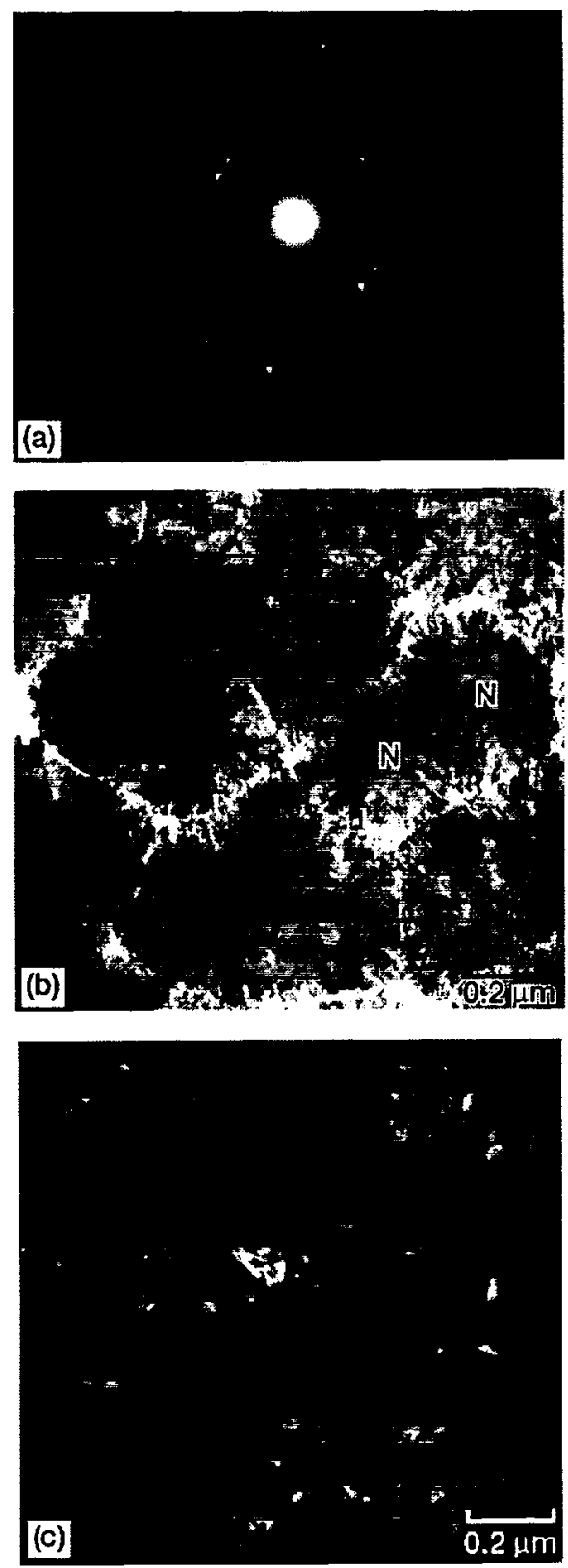

Figure 5.-Free-standing diamond films.

(a) Selected-area diffraction pattern.

(b) Bright-field TEM. (c) Dark-field TEM. 

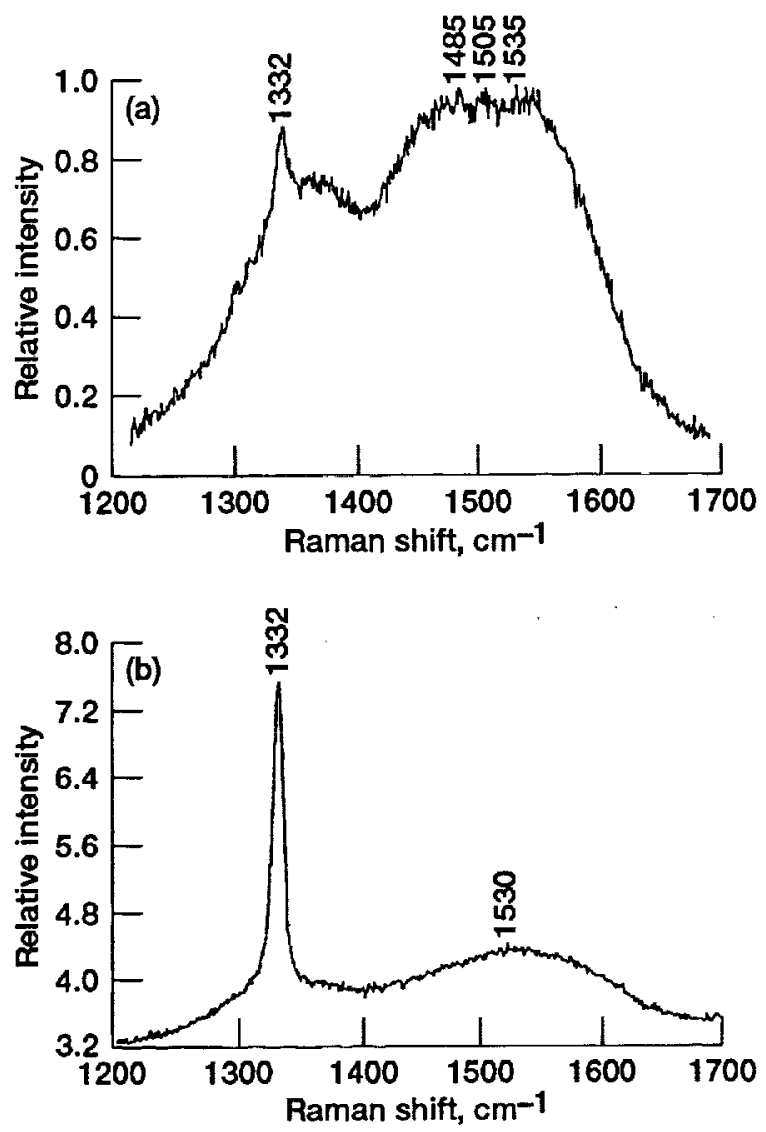

Figure 6.-Raman spectra of diamond films. (a) Fine-grain $(20$ to $100 \mathrm{~nm})$ diamond film on $\{100\}$ silicon substrate. (b) Medium-grain $(1100 \mathrm{~nm})$ diamond film on $\{100\}$ silicon substrate. 


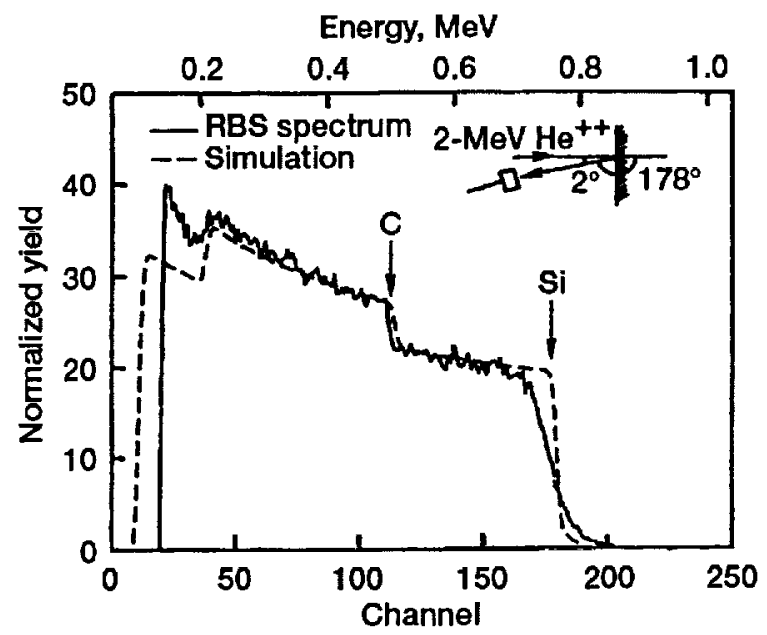

Figure 7.-Rutherford backscattering spectrum of fine-grain diamond film on silicon substrate. (Simulation curve was calculated by using the computer code RUMP.) 


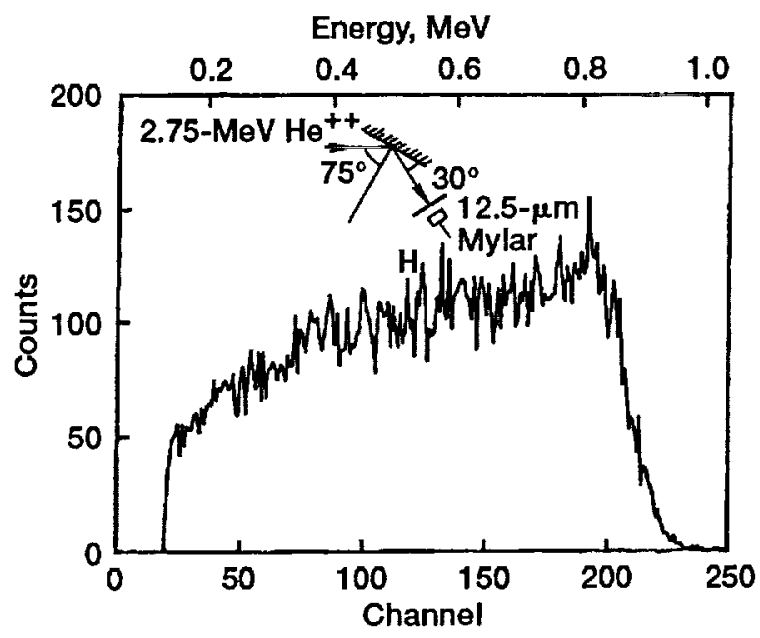

Figure 8.-Elastic recoil spectrum of fine-grain diamond film on sillicon substrate. 

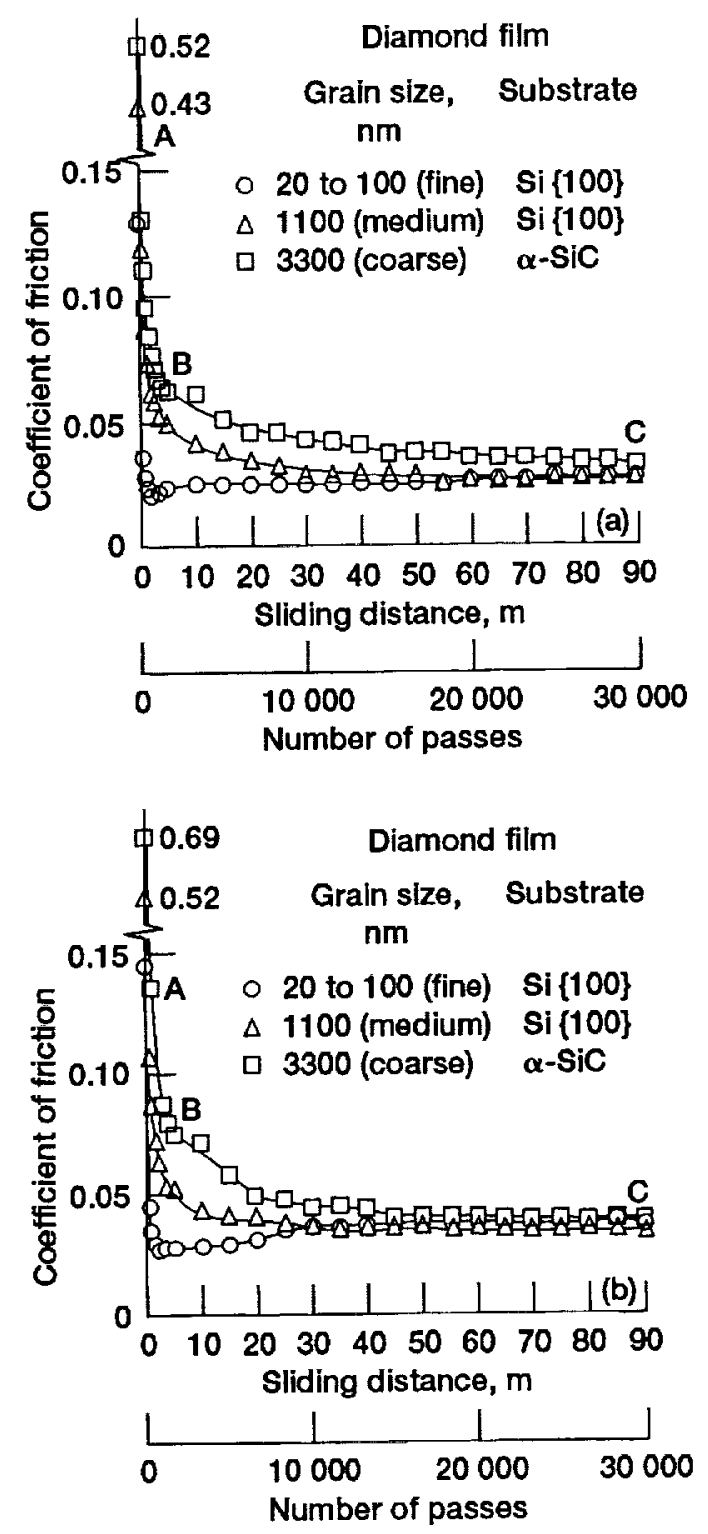

Figure 9.-Coefficient of friction as function of number of passes of bulk diamond pin in contact with fine-, medium-, and coarsegrain diamond films (a) in humid air (approx. $40 \%$ relative humidity) and (b) in dry nitrogen. 

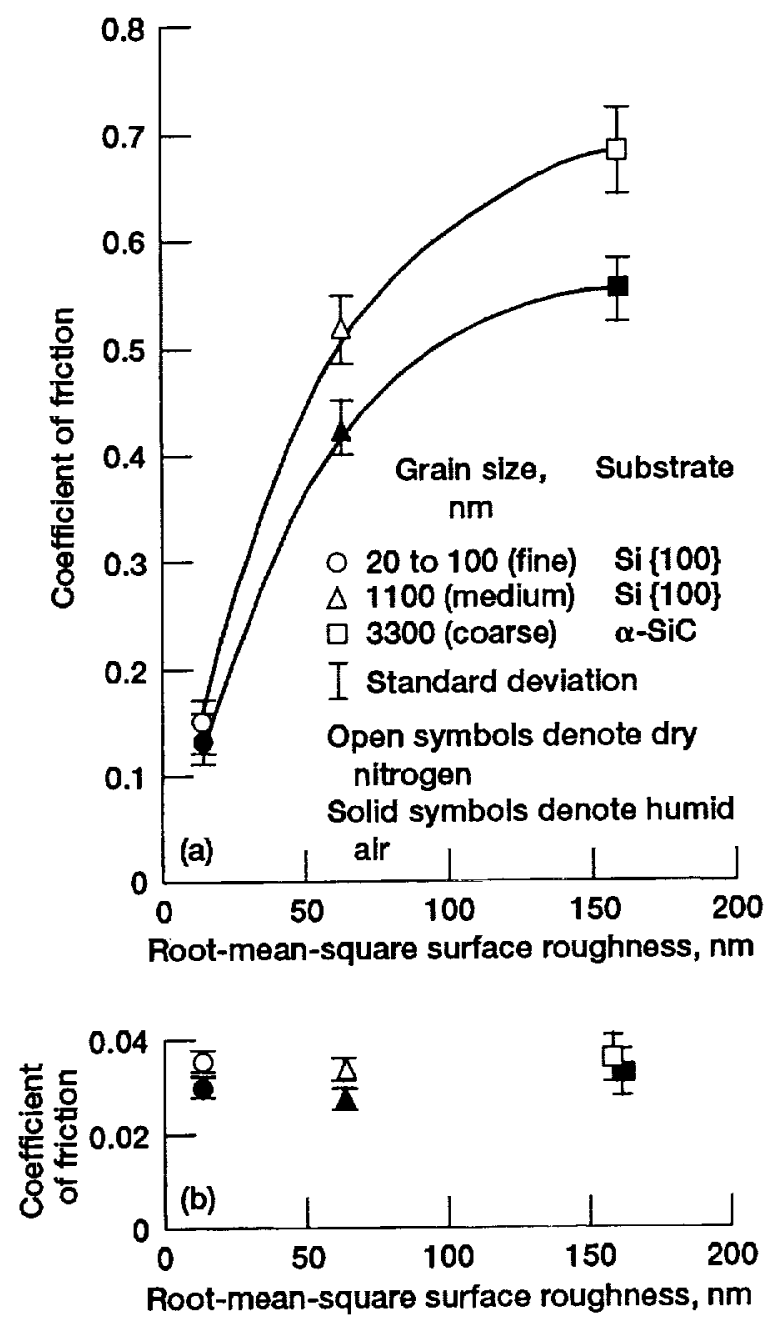

Figure 10.-Coefficient of friction as function of initial surface roughness of diamond films in humid air (approx. 40\% relative humidity) and in dry nitrogen. (a) Initial coefficients of friction. (b) Equilibrium coefficients of friction. 

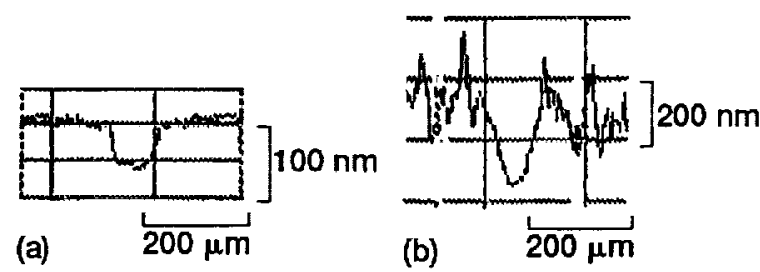

(c)

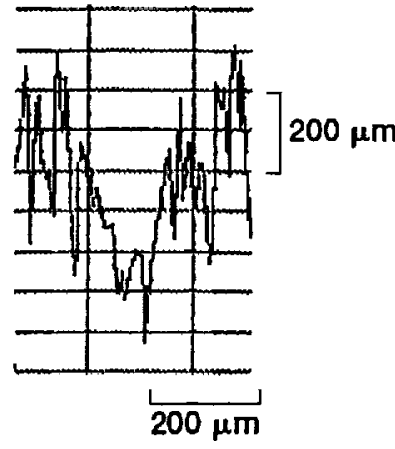

Figure 11.-Wear tracks (grooves) on diamond films after 30000 passes of bulk diamond pin in dry nitrogen. (a) Fine-grain (20 to $100 \mathrm{~nm}$ ) diamond film; Ims surface roughness, $15 \mathrm{~nm}$. (b) Medium-grain $(1100 \mathrm{~nm})$ diamond film; rms surface roughness, $63 \mathrm{~nm}$. (c) Coarse-grain ( $3300 \mathrm{~nm}$ ) diamond film; Ims surface roughness, $160 \mathrm{~nm}$. 


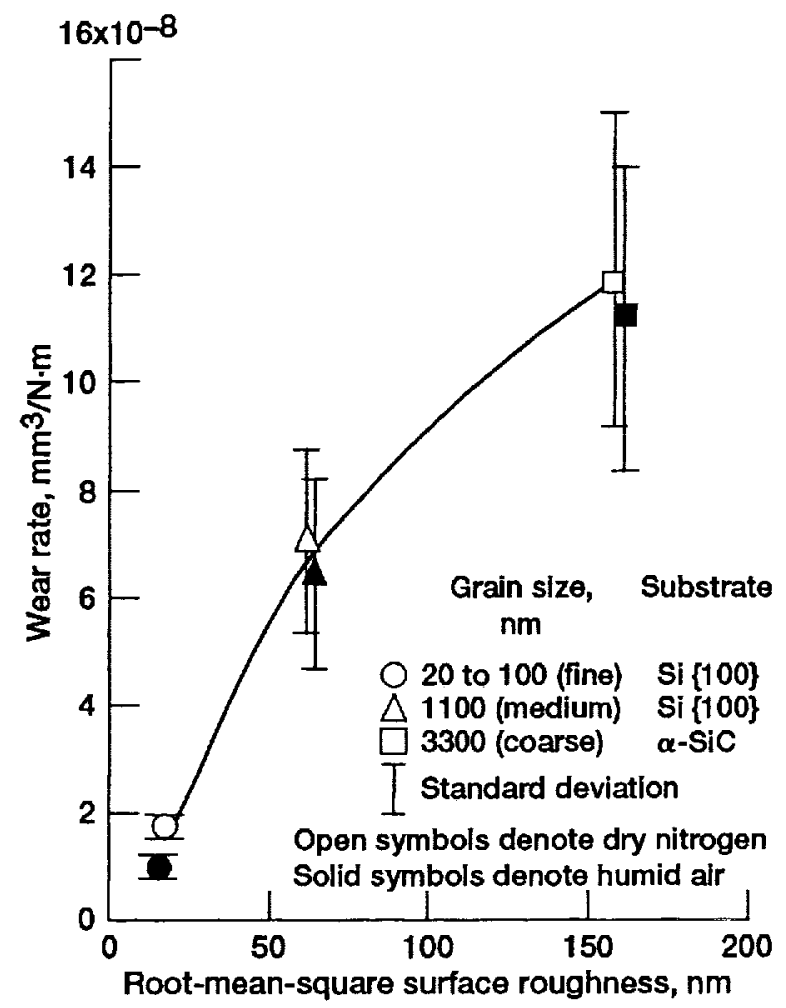

Figure 12.-Wear rate of diamond films as function of diamond surface roughness in humid air and in dry nitrogen. 

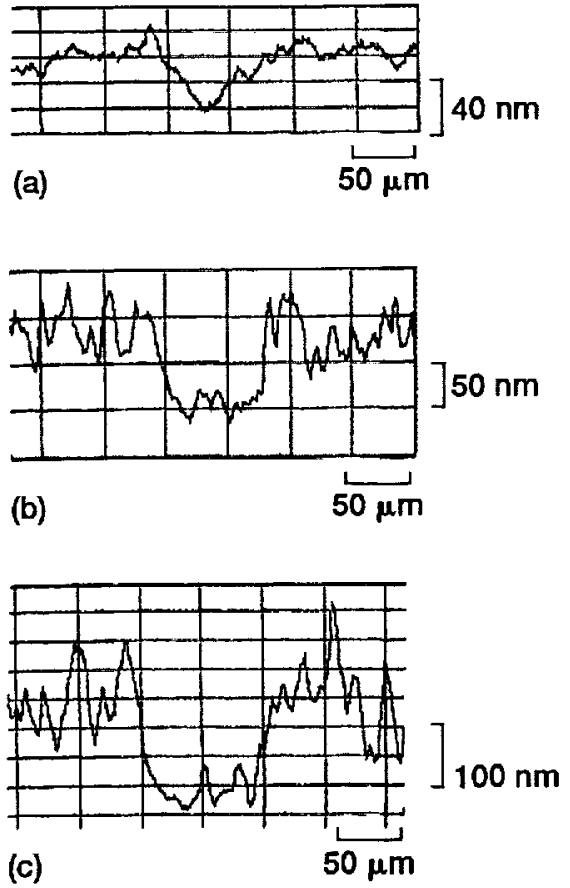

Figure 13.-Wear tracks (grooves) on dlamond films after 100 passes of bulk diamond pin in ultrahigh vacuum. (a) Fine-grain $(20$ to $100 \mathrm{~nm})$ diamond film on silicon substrate; rms surface roughness, $15 \mathrm{~nm}$. (b) Medium-grain (1000 nm) diamond film on silicon nitride substrate; $\mathrm{rms}$ surface roughness, $52 \mathrm{~nm}$. (c) Coarse-grain (1500 nm) diamond film on $\alpha$-silicon carbide substrate; rms surface roughness, $92 \mathrm{~nm}$. 


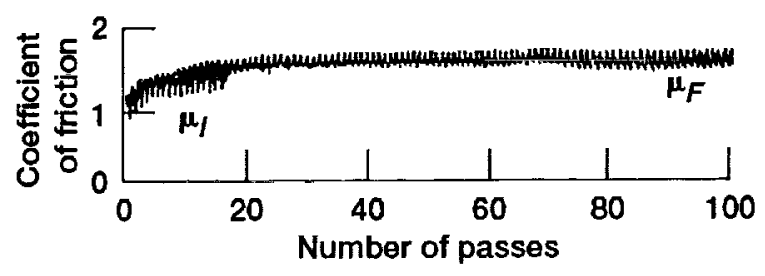

Figure 14.-Typical friction trace for bulk diamond pin in contact with diamond film on $\alpha$-silicon carbide substrate in ultrahigh vacuum (initial coefficient of friction, $\mu_{1}$; equilibrium coefficient of friction, $\left.\mu_{F}\right)$. 


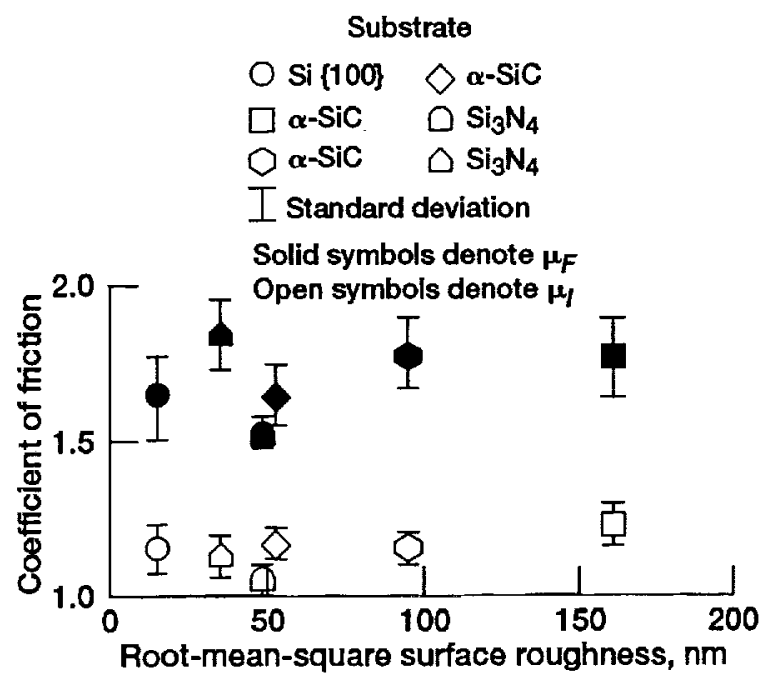

Figure 15.-Initial $\left(\mu_{l}\right)$ and equilibrium $\left(\mu_{F}\right)$ coefficients of friction as function of initial surface roughness of diamond films in ultrahigh vacuum. 


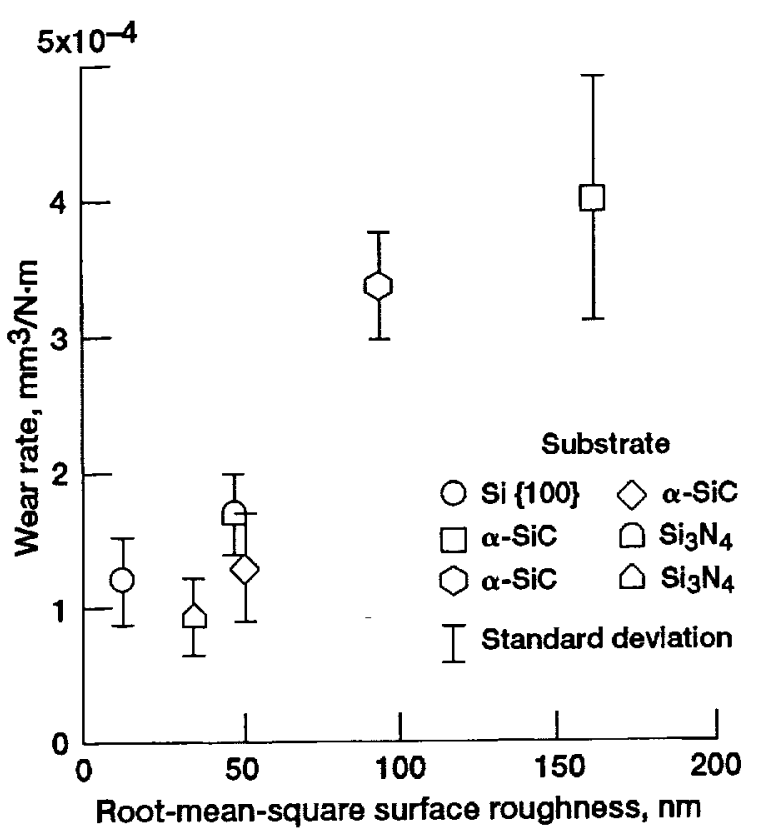

Figure 16.-Wear rate of diamond films in ultrahigh vacuum as function of initial surface roughness. 